\title{
Schultz and Modified Schultz Polynomials of Some Cog-Special Graphs
}

\author{
Ahmed M. Ali*, Haitham N. Mohammed \\ Department of Mathematics, University of Mosul, Mosul, Iraq \\ Email: *ahmed_math79@yahoo.com, haytham.nashwan@yahoo.com
}

How to cite this paper: Ali, A.M. and Mohammed, H.N. (2019) Schultz and Modified Schultz Polynomials of Some Cog-Special Graphs. Open Access Library Journal, 6: e5625.

https://doi.org/10.4236/oalib.1105625

Received: July 22, 2019

Accepted: August 24, 2019

Published: August 27, 2019

Copyright $\odot 2019$ by author(s) and Open Access Library Inc.

This work is licensed under the Creative Commons Attribution International License (CC BY 4.0).

http://creativecommons.org/licenses/by/4.0/

\section{(c) (i) Open Access}

\begin{abstract}
For a connected graph $G$, the Schultz and modified Schultz polynomials are defined as: $S c(G ; x)=\sum_{u, v \in V(G)}\left(\delta_{u}+\delta_{v}\right) x^{d(u, v)}$, and $\stackrel{*}{S} c^{*}(G ; x)=\sum_{u, v \in V(G)}\left(\delta_{u} \delta_{v}\right) x^{d(u, v)}$, respectively, where the summations are taken over all unordered pairs of distinct vertices in $V(G), \delta_{u}$ is the degree of vertex $u, d(u, v)$ is the distance between $u$ and $v$ and $V(G)$ is the vertex set of $G$. In this paper, we find Schultz and modified Schultz polynomials of the Cog-special graphs such as a complete graph, a star graph, a wheel graph, a path graph and a cycle graph. The Schultz index, modified Schultz index and average distance of Schultz and modified Schultz of each such Cog-special graphs are also obtained in this paper.
\end{abstract}

\section{Subject Areas}

Combinatorial Mathematics, Discrete Mathematics

\section{Keywords}

Cog-Special Graphs, Schultz and Modified Schultz Polynomials

\section{Introduction}

We follow the terminology of [1] [2] [3] [4]. All the graphs considered in this paper are simple and connected finite undirected without loops or multiple edges. Distance is an important concept in graph theory and it has applications to computer science, chemistry, and a variety of other fields [5] [6].

Suppose that $G=(V(G), E(G))$ is a simple undirected connected graph of order $p=p(G)=|V(G)|$ and size $q=q(G)=|E(G)|$, the distance between 
two vertices $u$ and $v$ of $G$ is denoted by $d(u, v)$ and it is defined as the length of a shortest $(u, v)$-path in connected graph $G$. In particular, if $u=v$, then $d(u, v)=0$. The greatest distance in $G$ is the diameter and will be denoted by $D$. The number of pairs of vertices of $G$ that are distance $k$ is denoted by $d(G, k)$. Let $D_{k}(G)$ be the set of all unordered pairs of vertices with distance $k$ such that $\left|D_{k}(G)\right|=d(G, k)$ and $\sum_{k=1}^{D} d(G, k)=\left(\begin{array}{l}p \\ 2\end{array}\right)$, where $\left(\begin{array}{l}p \\ 2\end{array}\right)$ is representation of the number of unordered pairs distinct vertices in $G$.

The Schultz polynomial of a graph $G$ is defined as:

$$
S c(G ; x)=\sum_{u, v \in V(G)}\left(\delta_{u}+\delta_{v}\right) x^{d(u, v)},
$$

and modified Schultz polynomial of a graph $G$ is defined as:

$$
\stackrel{*}{S c}(G ; x)=\sum_{u, v \in V(G)}\left(\delta_{u} \delta_{v}\right) x^{d(u, v)} .
$$

The molecular topological index (Schultz index) was introduced by Harry P. Schultz in 1993 [7] and the modified Schultz index was defined by S. Klavžar and

I. Gutman in 1997 [8].

The Schultz index is defined as:

$$
S c(G)=\sum_{u, v \in V(G)}\left(\delta_{u}+\delta_{v}\right) d(u, v),
$$

and modified Schultz index is defined as:

$$
\stackrel{*}{S} c(G)=\sum_{u, v \in V(G)}\left(\delta_{u} \delta_{v}\right) d(u, v) .
$$

where the summation for all above is taken over all unordered pairs of distinct vertices in $V(G)$.

The indices of Schultz and modified Schultz can be obtained by the derivative of Schultz and modified Schultz polynomials with respect to $x$ at $x=1$, i.e.:

$$
S c(G)=\left.\frac{\mathrm{d}}{\mathrm{d} x} \operatorname{Sc}(G ; x)\right|_{x=1} \text {, and } \stackrel{*}{S} c(G)=\left.\frac{\mathrm{d}}{\mathrm{d} x} \stackrel{*}{S} c(G ; x)\right|_{x=1} \text { respectively. }
$$

The average distance of a connected graph $G$ with respect Schultz and modified Schultz is defined as:

$$
\overline{S c(G)}=\frac{S c(G)}{\left(\begin{array}{l}
p \\
2
\end{array}\right)} \text { and } \overline{S_{c}(G)}=\frac{{ }_{S c}^{*}(G)}{\left(\begin{array}{l}
p \\
2
\end{array}\right)} \text {. }
$$

Schultz and modified Schultz polynomial of two operations Gutman's and the Cog-complete bipartite Graphs founded by Ahmed and Haitham [9] [10], the Schultz and modified Schultz polynomial of some special graphs are summarized in the following theorem (See [11]).

Theorem 1.1:

1) $S c\left(K_{p} ; x\right)=p(p-1)^{2} x^{1}, \quad S_{C}^{*}\left(K_{p} ; x\right)=\left\{p(p-1)^{3} / 2\right\} x^{1}$. 
2) $\operatorname{Sc}\left(S_{p+1} ; x\right)=p(p+1) x^{1}+p(p-1) x^{2}$,

$S^{*} c\left(S_{p+1} ; x\right)=p^{2} x^{1}+\{p(p-1) / 2\} x^{2}$.

3) $\operatorname{Sc}\left(W_{p+1} ; x\right)=\left(p^{2}+9 p+6\right) x^{1}+3 p(p-3) x^{2}$,

${ }^{*} c\left(W_{p+1} ; x\right)=3\left(p^{2}+3 p+3\right) x^{1}+\{9 p(p-3) / 2\} x^{2}$.

4) $S c\left(P_{p} ; x\right)=\sum_{k=1}^{p-1}[4(p-k)-2] x^{k}, \quad S^{*}\left(P_{p} ; x\right)=4 \sum_{k=1}^{p-1}(p-k-1) x^{k}+x^{p-1}$.

5) $S c\left(C_{p} ; x\right)=\stackrel{*}{S} c\left(C_{p} ; x\right)=4 p \sum_{k=1}^{\lceil p / 2\rceil-1} x^{k}+\left[\begin{array}{ll}2 p x^{p / 2}, & p \text { is even, } \\ 0, & p \text { is odd. }\end{array}\right.$

\section{Main Results}

\subsection{Definition}

A cog-complete graph $K_{p}^{c}$ is the graph constructed from a complete graph $K_{p}$, $p \geq 3$, of vertex set $\left\{u_{1}, u_{2}, \cdots, u_{p}\right\}$ with $p$ additional vertices $\left\{v_{1}, v_{2}, \cdots, v_{p}\right\}$, and 2p edges $\left\{v_{i} u_{i}, v_{i} u_{i+1}: i=1,2, \cdots, p\right\}, \quad\left(u_{p+1} \equiv u_{1}\right)$, as shown in Figure 1 .

It is clear that $p\left(K_{p}^{c}\right)=2 p, q\left(K_{p}^{c}\right)=p(p+3) / 2$, and $\operatorname{diam} K_{p}^{c}=3$, for $p \geq 4$.

Theorem 2.1.1: For $p \geq 4$, we have:

1) $S c\left(K_{p}^{c} ; x\right)=p\left(p^{2}+2 p+5\right) x^{1}+p(p-1)(p+2) x^{2}+2 p(p-3) x^{3}$.

2) $S^{*} c\left(K_{p}^{c} ; x\right)=\left\{p(p+1)\left(p^{2}+7\right) / 2\right\} x^{1}+2 p^{2}(p-1) x^{2}+2 p(p-3) x^{3}$.

Proof: For every vertice $y, z \in V\left(K_{p}^{c}\right)$, there is $d(y, z)=k, k=1,2,3$, and obviously $\sum_{i=1}^{3}\left|D_{i}\right|=p(2 p-1)$.

We will have three partitions for proof:

P1. If $d(y, z)=1$, then $\left|D_{1}\right|=p(p+3) / 2$ and is equal to $q\left(K_{p}^{c}\right)$, we have two subsets of it:

P1.1.

$$
\mid\left\{\left(u_{i}, v_{j}\right): u_{i} v_{j} \in E\left(K_{p}^{c}\right), \delta_{u_{i}}+\delta_{v_{j}}=p+3 \& \delta_{u_{i}} \delta_{v_{j}}=2(p+1),\right.
$$

$$
\left.i=j, j+1,1 \leq j \leq p,\left(u_{p+1} \equiv u_{1}\right)\right\} \mid=2 p \text {. }
$$

P1.2.

$$
\mid\left\{\left(u_{i}, u_{j}\right): u_{i} u_{j} \in E\left(K_{p}^{c}\right), \delta_{u_{i}}+\delta_{u_{j}}=2(p+1)\right.
$$

$$
\left.\& \delta_{u_{i}} \delta_{u_{j}}=(p+1)^{2}, 1 \leq i, j \leq p, i \neq j\right\} \mid=p(p-1) / 2 .
$$

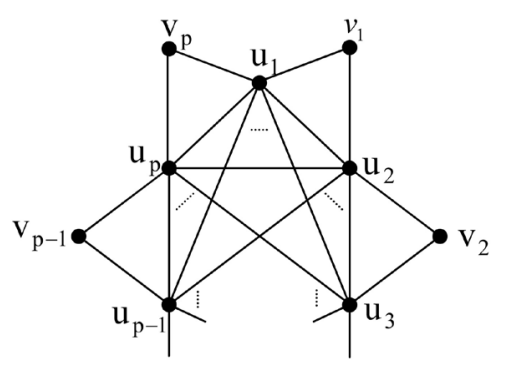

Figure 1. A cog-complete graph $K_{p}^{c}$. 
P2. If $d(y, z)=2$, then $\left|D_{2}\right|=p(p-1)$, we have two subsets of it:

P2.1. $\left|\left\{\left(v_{i}, v_{i+1}\right): \delta_{v_{i}}+\delta_{v_{i+1}}=4 \& \delta_{v_{i}} \delta_{v_{i+1}}=4,1 \leq i \leq p,\left(v_{p+1} \equiv v_{1}\right)\right\}\right|=p$.

P2.2. $\left\{\left\{\left(v_{i}, u_{j}\right): v_{i} u_{j} \notin E\left(K_{p}^{c}\right), \delta_{v_{i}}+\delta_{u_{j}}=p+3 \& \delta_{v_{i}} \delta_{u_{j}}=2(p+1)\right.\right.$,

$$
\left.1 \leq i, j \leq p, i \neq j, j+1\left(u_{p+1} \equiv u_{1}\right)\right\} \mid=p(p-2) \text {. }
$$

P3. If $d(y, z)=3$, then $\left|D_{3}\right|=p(p-3) / 2$, we have:

$$
\begin{aligned}
& \left|\left\{\left(v_{i}, v_{j}\right): \delta_{v_{i}}+\delta_{v_{j}}=4 \& \delta_{v_{i}} \delta_{v_{j}}=4,1 \leq i, j \leq p,|i-j| \neq 0,1\right\}\right|-\left|\left\{\left(v_{1}, v_{p}\right)\right\}\right| \\
& =p(p-3) / 2 .
\end{aligned}
$$

From P1 - P3, we have:

$$
\begin{aligned}
& S c\left(K_{p}^{c} ; x\right)=p\left(p^{2}+2 p+5\right) x^{1}+p(p-1)(p+2) x^{2}+2 p(p-3) x^{3} . \\
& S^{*} c\left(K_{p}^{c} ; x\right)=\left\{p(p+1)\left(p^{2}+7\right) / 2\right\} x^{1}+2 p^{2}(p-1) x^{2}+2 p(p-3) x^{3} .
\end{aligned}
$$

Corollary 2.1.2: For $p \geq 4$, we have:

1) $S c\left(K_{p}^{c}\right)=p\left(3 p^{2}+10 p-17\right)$.

2) $\stackrel{*}{S} c\left(K_{p}^{c}\right)=p\left(p^{3}+9 p^{2}+11 p-29\right) / 2$.

Corollary 2.1.3: For $p \geq 4$, we have:

1) $10 \frac{1}{7} \leq \overline{S c}\left(K_{p}^{c}\right)<(6 p+23) / 4$.

2) $15 \frac{13}{14} \leq \overline{S^{*}} c\left(K_{p}^{c}\right)<\left(4 p^{2}+38 p+63\right) / 16$.

\section{Remark 2.1.4:}

1) $S_{c}\left(K_{3}^{c} ; x\right)=60 x^{1}+30 x^{2}$.

2) $S_{c}^{*}\left(K_{3}^{c} ; x\right)=96 x^{1}+36 x^{2}$.

\subsection{Definition}

A cog-star graph $S_{p+1}^{c}$ is the graph constructed from a star graph, $S_{p+1}, p \geq 3$, of vertex set $\left\{u_{0}, u_{1}, \cdots, u_{p-1}, u_{p}\right\}$ with $p$ additional vertices $\left\{v_{1}, v_{2}, \cdots, v_{p-1}, v_{p}\right\}$, and edges $\left\{v_{i} u_{i}, v_{i} u_{i+1}: i=1,2, \cdots, p\right\},\left(u_{p+1} \equiv u_{1}\right)$, as shown in Figure 2 .

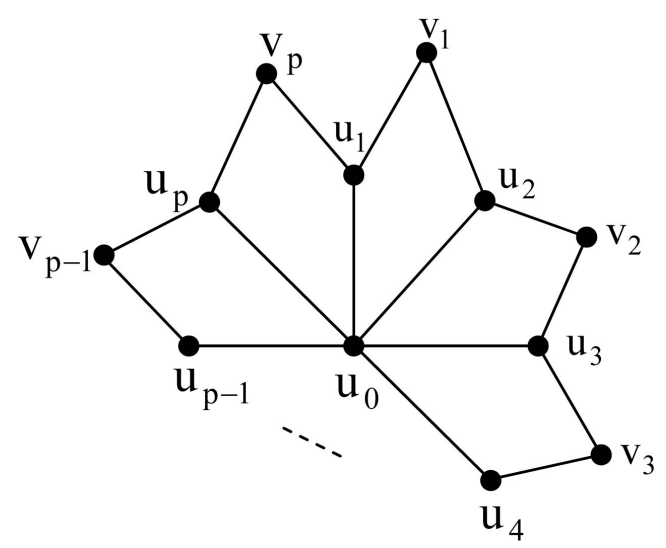

Figure 2. A cog-star graph $S_{p+1}^{c}$. 
It is clear that $p\left(S_{p+1}^{c}\right)=2 p+1, q\left(S_{p+1}^{c}\right)=3 p, \operatorname{diam} S_{p+1}^{c}=4$ for $p \geq 4$.

Theorem 2.2.1: For $p \geq 4$, we have:

1) $S c\left(S_{p+1}^{c} ; x\right)=p(p+13) x^{1}+p(4 p+3) x^{2}+5 p(p-2) x^{3}+2 p(p-3) x^{4}$.

2) $S^{*} c\left(S_{p+1}^{c} ; x\right)=3 p(p+4) x^{1}+\{p(13 p-1) / 2\} x^{2}+6 p(p-2) x^{3}+2 p(p-3) x^{4}$

Proof: For every vertice $y, z \in V\left(S_{p+1}^{c}\right)$, there is $d(y, z)=k, k=1,2,3,4$, and obviously $\sum_{i=1}^{4}\left|D_{i}\right|=p(2 p+1)$.

We will have four partitions for proof:

P1. If $d(y, z)=1$, then $\left|D_{1}\right|=3 p$ and is equal to $q\left(S_{p+1}^{c}\right)$, we have two subsets of it:

P1.1. $\left|\left\{\left(u_{0}, u_{i}\right): u_{0} u_{i} \in E\left(S_{p+1}^{c}\right), \delta_{u_{0}}+\delta_{u_{i}}=p+3 \& \delta_{u_{0}} \delta_{u_{i}}=3 p, 1 \leq i \leq p\right\}\right|=p$. $\left\{\left\{\left(v_{i}, u_{j}\right): v_{i} u_{j} \in E\left(S_{p+1}^{c}\right), \delta_{v_{i}}+\delta_{u_{j}}=5 \& \delta_{v_{i}} \delta_{u_{j}}=6,1 \leq i \leq p\right.\right.$, $\left.j=i, i+1,\left(u_{p+1} \equiv u_{1}\right)\right\} \mid=2 p$.

P2. If $d(y, z)=2$, then $\left|D_{2}\right|=p(p+3) / 2$, we have three subsets:

P2.1. $\left|\left\{\left(u_{i}, u_{j}\right): \delta_{u_{i}}+\delta_{u_{j}}=6 \& \delta_{u_{i}} \delta_{u_{j}}=9,1 \leq i, j \leq p, i \neq j\right\}\right|=p(p-1) / 2$.

P2.2. $\left|\left\{\left(u_{0}, v_{i}\right): \delta_{u_{0}}+\delta_{v_{i}}=p+2 \& \delta_{u_{0}} \delta_{v_{i}}=2 p, 1 \leq i \leq p\right\}\right|=p$.

P2.3. $\left|\left\{\left(v_{i}, v_{i+1}\right): \delta_{v_{i}}+\delta_{v_{i+1}}=4 \& \delta_{v_{i}} \delta_{v_{i+1}}=4,1 \leq i \leq p,\left(v_{p+1} \equiv v_{1}\right)\right\}\right|=p$.

P3. If $d(y, z)=3$, then $\left|D_{3}\right|=p(p-2)$, we have:

$$
\begin{aligned}
& \left|\left\{\left(u_{i}, v_{j}\right): \delta_{u_{i}}+\delta_{v_{j}}=5 \& \delta_{u_{i}} \delta_{v_{j}}=6,1 \leq i, j \leq p, i-j \neq 0,1\right\}-\left\{\left(u_{1}, v_{p}\right)\right\}\right| \\
& =p(p-2) .
\end{aligned}
$$

P4. If $d(y, z)=4$, then $\left|D_{4}\right|=p(p-3) / 2$, we have:

$$
\begin{aligned}
& \left|\left\{\left(v_{i}, v_{j}\right): \delta_{v_{i}}+\delta_{v_{j}}=4 \& \delta_{v_{i}} \delta_{v_{j}}=4,1 \leq i \leq p-2, i+2 \leq j \leq p\right\}-\left\{\left(v_{1}, v_{p}\right)\right\}\right| \\
& =p(p-3) / 2 .
\end{aligned}
$$

From P1 - P4, we have:

$$
\begin{gathered}
S c\left(S_{p+1}^{c} ; x\right)=p(p+13) x^{1}+p(4 p+3) x^{2}+5 p(p-2) x^{3}+2 p(p-3) x^{4} . \\
S_{c}^{*}\left(S_{p+1}^{c} ; x\right)=3 p(p+4) x^{1}+\{p(13 p-1) / 2\} x^{2}+6 p(p-2) x^{3}+2 p(p-3) x^{4} .
\end{gathered}
$$

Corollary 2.2.2: For $p \geq 4$, we have:

1) $S c\left(S_{p+1}^{c}\right)=p(32 p-35)$.

2) $S^{*} c\left(S_{p+1}^{c}\right)=7 p(6 p-7)$.

Corollary 2.2.3: For $p \geq 4$, we have:

1) $10 \frac{1}{3} \leq \overline{S c}\left(S_{p+1}^{c}\right)<16$.

2) $13 \frac{2}{9} \leq \overline{S^{*}} c\left(S_{p+1}^{c}\right)<21$.

\section{Remark 2.2.3:}


1) $\operatorname{Sc}\left(S_{4}^{c} ; x\right)=48 x^{1}+45 x^{2}+15 x^{3}$.

2) $S_{c}^{*}\left(S_{4}^{c} ; x\right)=63 x^{1}+57 x^{2}+18 x^{3}$.

\subsection{Definition}

A cog-wheel graph $W_{p+1}^{c}$ is the graph constructed from a wheel $W_{p+1}, p \geq 3$, of order $p+1$, with vertex set $\left\{u_{0}, u_{1}, u_{2}, \cdots, u_{p}\right\}$ and with $p$ additional vertices $v_{1}, v_{2}, \cdots, v_{p}$, and edges $\left\{v_{i} u_{i}, v_{i} u_{i+1}: i=1,2, \cdots, p\right\},\left(u_{p+1} \equiv u_{1}\right)$, as shown in Figure 3.

It is clear that $p\left(W_{p+1}^{c}\right)=2 p+1, q\left(W_{p+1}^{c}\right)=4 p, \operatorname{diam} W_{p+1}^{c}=4$ for $p \geq 6$.

Theorem 2.3.1: For $p \geq 6$, we have:

1) $S c\left(W_{p+1}^{c} ; x\right)=p(p+29) x^{1}+p(6 p+5) x^{2}+p(7 p-24) x^{3}+2 p(p-5) x^{4}$.

2) ${ }^{*} c\left(W_{p+1}^{c} ; x\right)=5 p(p+9) x^{1}+\{p(29 p-27) / 2\} / x^{2}+2 p(5 p-18) x^{3}$

$$
+2 p(p-5) x^{4} \text {. }
$$

Proof: For every vertice $y, z \in V\left(W_{p+1}^{c}\right)$, there is $d(y, z)=k, k=1,2,3,4$, and obviously $\sum_{i=1}^{4}\left|D_{i}\right|=p(2 p+1)$.

We will have four partitions for proof:

P1. If $d(y, z)=1$, then $\left|D_{1}\right|=4 p$ and is equal to $q\left(W_{p+1}^{c}\right)$, we have three subsets of it:

P1.1. $\left|\left\{\left(u_{0}, u_{i}\right): u_{0} u_{i} \in E\left(W_{p+1}^{c}\right), \delta_{u_{0}}+\delta_{u_{i}}=p+5 \& \delta_{u_{0}} \delta_{u_{i}}=5 p, 1 \leq i \leq p\right\}\right|=p$.

P1.2.

$\left\{\left(u_{i}, u_{i+1}\right): u_{i} u_{i+1} \in E\left(W_{p+1}^{c}\right), \delta_{u_{i}}+\delta_{u_{i+1}}=10\right.$

$\left.\& \delta_{u_{i}} \delta_{u_{i+1}}=25,1 \leq i \leq p,\left(u_{p+1} \equiv u_{1}\right)\right\} \mid=p$.

P1.3.

$\mid\left\{\left(v_{i}, u_{j}\right): v_{i} u_{j} \in E\left(W_{p+1}^{c}\right), \delta_{v_{i}}+\delta_{u_{j}}=7\right.$

$$
\left.\& \delta_{v_{i}} \delta_{u_{j}}=10,1 \leq i \leq p, j=i, i+1,\left(u_{p+1} \equiv u_{1}\right)\right\} \mid=2 p .
$$

P2. If $d(y, z)=2$, then $\left|D_{2}\right|=p(p+5) / 2$, we have five subsets

P2.1. $\left\{\left\{\left(u_{i}, u_{j}\right): \delta_{u_{i}}+\delta_{u_{j}}=10 \& \delta_{u_{i}} \delta_{u_{j}}=25,1 \leq i \leq p-2, i+2 \leq j \leq p\right\}\right.$ $-\left\{\left(u_{1}, u_{p}\right)\right\} \mid=p(p-3) / 2$.

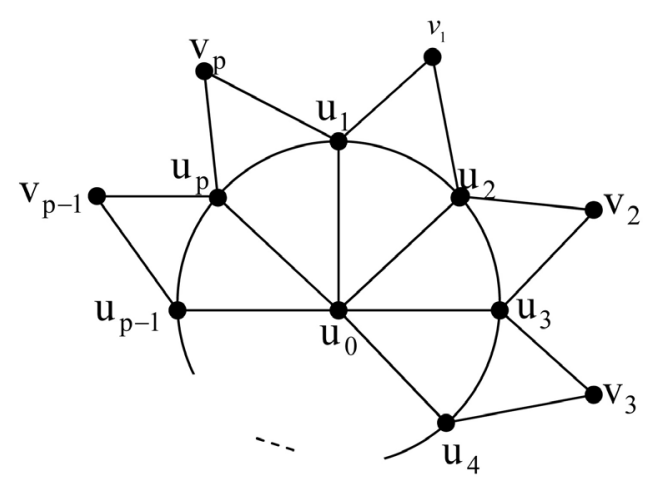

Figure 3. A cog-wheel graph $W_{p+1}^{c}$. 
P2.2. $\left|\left\{\left(u_{0}, v_{i}\right): \delta_{u_{0}}+\delta_{v_{i}}=p+2 \& \delta_{u_{0}} \delta_{v_{i}}=2 p, 1 \leq i \leq p\right\}\right|=p$.

P2.3. $\left|\left\{\left(u_{i}, v_{i+1}\right): \delta_{u_{i}}+\delta_{v_{i+1}}=7 \& \delta_{u_{i}} \delta_{v_{i+1}}=10,1 \leq i \leq p,\left(v_{p+1} \equiv v_{1}\right)\right\}\right|=p$.

P2.4. $\left\{\left(u_{i}, v_{i-2}\right): \delta_{u_{i}}+\delta_{v_{i-2}}=7 \& \delta_{u_{i}} \delta_{v_{i-2}}=10,3 \leq i \leq p\right\}$

$$
\bigcup\left\{\left(u_{1}, v_{p-1}\right),\left(u_{2}, v_{p}\right)\right\} \mid=p .
$$

P2.5. $\left|\left\{\left(v_{i}, v_{i+1}\right): \delta_{v_{i}}+\delta_{v_{i+1}}=4 \& \delta_{v_{i}} \delta_{v_{i+1}}=4,1 \leq i \leq p,\left(v_{p+1} \equiv v_{1}\right)\right\}\right|=p$.

P3. If $d(y, z)=3$, then $\left|D_{3}\right|=p(p-3)$, we have two subsets:

$$
\mid\left\{\left(u_{i}, v_{j}\right): \delta_{u_{i}}+\delta_{v_{j}}=7 \& \delta_{u_{i}} \delta_{v_{j}}=10,3 \leq i \leq p, 1 \leq j \leq p, j \neq i-2, i-1,\right.
$$

P3.1. $\left.i, i+1,\left(v_{p+1} \equiv v_{1}\right)\right\} \cup\left\{\left(u_{i}, v_{j}\right): i=1,2, i+2 \leq j \leq p+i-3\right\} \mid$

$$
=p(p-4) \text {. }
$$

P3.2.

$$
\left\{\left(v_{i}, v_{i+2}\right): \delta_{v_{i}}+\delta_{v_{i+2}}=4 \& \delta_{v_{i}} \delta_{v_{i+2}}=4,1 \leq i \leq p,\left(v_{p+1} \equiv v_{1}\right),\left(v_{p+2} \equiv v_{2}\right)\right\} \mid=p .
$$

P4. If $d(y, z)=4$, then $\left|D_{4}\right|=p(p-5) / 2$, we have:

$$
\begin{aligned}
& \mid\left\{\left(v_{i}, v_{j}\right): \delta_{v_{i}}+\delta_{v_{j}}=4 \& \delta_{v_{i}} \delta_{v_{j}}=4,3 \leq i \leq p, 1 \leq j \leq p, j \neq i-2, i-1, i, i+1,\right. \\
& \left.i+2,\left(v_{p+1} \equiv v_{1}\right),\left(v_{p+2} \equiv v_{2}\right)\right\} \cup\left\{\left(v_{i}, v_{j}\right): i=1,2, i+3 \leq j \leq p+i-3\right\} \mid \\
& =p(p-5) / 2 .
\end{aligned}
$$

From P1 - P4, we have:

$$
\begin{aligned}
S c\left(W_{p+1}^{c} ; x\right)=p( & p+29) x^{1}+p(6 p+5) x^{2}+p(7 p-24) x^{3}+2 p(p-5) x^{4} . \\
S_{c}^{*}\left(W_{p+1}^{c} ; x\right)= & 5 p(p+9) x^{1}+\{p(29 p-27) / 2\} x^{2}+2 p(5 p-18) x^{3} \\
& +2 p(p-5) x^{4} .
\end{aligned}
$$

Corollary 2.3.2: For $p \geq 6$, we have:

1) $S c\left(W_{p+1}^{c}\right)=p(42 p-73)$.

2) ${ }^{*} c\left(W_{p+1}^{c}\right)=2 p(36 p-65)$.

Corollary 2.3.3: For $p \geq 6$, we have:

1) $13 \frac{10}{13} \leq \overline{S c}\left(W_{p+1}^{c}\right)<21$.

2) $23 \frac{3}{13} \leq \overline{S^{*}} c\left(W_{p+1}^{c}\right)<36$.

\section{Remark 2.3.4:}

1) $S c\left(W_{6}^{c} ; x\right)=170 x^{1}+175 x^{2}+55 x^{3}, S c\left(W_{5}^{c} ; x\right)=132 x^{1}+116 x^{2}+8 x^{3}$, $\operatorname{Sc}\left(W_{4}^{c} ; x\right)=96 x^{1}+48 x^{2}$.

2) $S^{*} c\left(W_{6}^{c} ; x\right)=350 x^{1}+295 x^{2}+70 x^{3}, \quad \stackrel{*}{S c}\left(W_{5}^{c} ; x\right)=260 x^{1}+178 x^{2}+8 x^{3}$, $S^{*} c\left(W_{4}^{c} ; x\right)=180 x^{1}+60 x^{2}$. 


\subsection{Definition}

A saw graph $P_{p}^{c}$ is a path of order $p$, say $\left\{u_{1}, u_{2}, \cdots, u_{p}\right\}$, with $p-1$ additional vertices $\left\{v_{1}, v_{2}, \cdots, v_{p-1}\right\}$ and edges $\left\{v_{i} u_{i}, v_{i} u_{i+1}: i=1,2, \cdots, p-1\right\}$ as depicted in Figure 4.

It is clear $p\left(P_{p}^{c}\right)=2 p-1, q\left(P_{p}^{c}\right)=3(p-1)$ and $\operatorname{diam} P_{p}^{c}=p-1$, for $p \geq 2$.

Theorem 2.4.1: For $p \geq 5$, we have:

1) $S c\left(P_{p}^{c} ; x\right)=4(5 p-7) x^{1}+8 \sum_{k=2}^{p-3}(3 p-3 k-1) x^{k}+40 x^{p-2}+16 x^{p-1}$.

2) $\stackrel{*}{S}^{*}\left(P_{p}^{c} ; x\right)=8(4 p-7) x^{1}+12 \sum_{k=2}^{p-3}(3 p-3 k-2) x^{k}+48 x^{p-2}+16 x^{p-1}$.

\section{Proof:}

For every vertice $y, z \in V\left(P_{p}^{c}\right)$, there is $d(y, z)=k, 1 \leq k \leq p-1$, and obviously $\sum_{i=1}^{p-1}\left|D_{i}\right|=(2 p-1)(p-1)$.

We will have four partitions for proof:

P1. if $d(y, z)=1$, then $\left|D_{1}\right|=3(p-1)$ and is equal to $q\left(P_{p}^{c}\right)$, we have five subsets of it:

P1.1. $\left|\left\{\left(u_{i}, u_{i+1}\right): u_{i} u_{i+1} \in E\left(P_{p}^{c}\right), \delta_{u_{i}}+\delta_{u_{i+1}}=6, \delta_{u_{i}} \delta_{u_{i+1}}=8, i=1, p-1\right\}\right|=2$.

P1.2. $\mid\left\{\left(u_{1}, v_{1}\right),\left(u_{p}, v_{p-1}\right): u_{1} v_{1}, u_{p} v_{p-1} \in E\left(P_{p}^{c}\right), \delta_{u_{1}\left(u_{p}\right)}+\delta_{v_{1}\left(v_{p-1}\right)}=4\right.$

$$
\left.\delta_{u_{1}\left(u_{p}\right)} \delta_{v_{1}\left(v_{p-1}\right)}=4\right\} \mid=2 \text {. }
$$

P1.3. $\left|\left\{\left(u_{i}, v_{i-1}\right): u_{i} v_{i-1} \in E\left(P_{p}^{c}\right), \delta_{u_{i}}+\delta_{v_{i-1}}=6, \delta_{u_{i}} \delta_{v_{i-1}}=8,2 \leq i \leq p-1\right\}\right|=p-2$.

P1.4. $\left|\left\{\left(u_{i}, v_{i}\right): u_{i} v_{i} \in E\left(P_{p}^{c}\right), \delta_{u_{i}}+\delta_{v_{i}}=6, \delta_{u_{i}} \delta_{v_{i}}=8,2 \leq i \leq p-1\right\}\right|=p-2$.

\section{P1.5.}

$$
\left|\left\{\left(u_{i}, u_{i+1}\right): u_{i} u_{i+1} \in E\left(P_{p}^{c}\right), \delta_{u_{i}}+\delta_{u_{i+1}}=8, \delta_{u_{i}} \delta_{u_{i+1}}=16,2 \leq i \leq p-2\right\}\right|=p-3 .
$$

P2. if $d(y, z)=k, 2 \leq k \leq p-3$, then $\sum_{k=2}^{p-3}\left|D_{k}\right|=2(p-4)(p+1)$, we have six subsets of it:

P2.1.

$$
\begin{aligned}
& \mid\left\{\left(u_{1}, u_{k+1}\right): \delta_{u_{1}}+\delta_{u_{k+1}}=6, \delta_{u_{1}} \delta_{u_{k+1}}=8\right\} \\
& \cup\left\{\left(u_{1}, v_{k}\right): \delta_{u_{1}}+\delta_{v_{k}}=4, \delta_{u_{1}} \delta_{v_{k}}=4\right\} \mid=2 . \\
& \mid\left\{\left(u_{p}, u_{p-k}\right): \delta_{u_{p}}+\delta_{u_{p-k}}=6, \delta_{u_{p}} \delta_{u_{p-k}}=8\right\}
\end{aligned}
$$

P2.2.

$$
\bigcup\left\{\left(u_{p}, v_{p-k}\right): \delta_{u_{p}}+\delta_{v_{p-k}}=4, \delta_{u_{p}} \delta_{v_{p-k}}=4\right\} \mid=2 .
$$

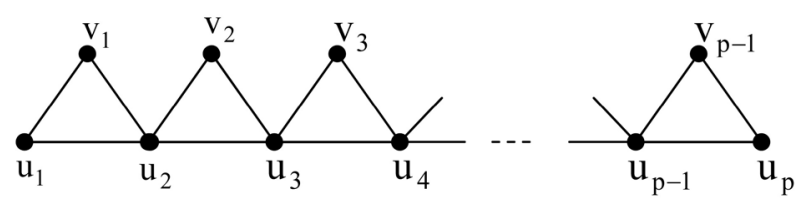

Figure 4. A saw graph $P_{p}^{c}$. 
P2.3. $\left|\left\{\left(u_{i}, u_{k+i}\right): \delta_{u_{i}}+\delta_{u_{k+i}}=8, \delta_{u_{i}} \delta_{u_{k+i}}=16,2 \leq i \leq p-k-1\right\}\right|$

$$
=p-k-2 \text {. }
$$

P2.4. $\left|\left\{\left(u_{i}, v_{k+i-1}\right): \delta_{u_{i}}+\delta_{v_{k+i-1}}=6, \delta_{u_{i}} \delta_{v_{k+i-1}}=8,2 \leq i \leq p-k\right\}\right|=p-k-1$.

P2.5. $\left|\left\{\left(u_{p-i+1}, v_{p-k-i+1}\right): \delta_{u_{p-i+1}}+\delta_{v_{p-k-i+1}}=6, \delta_{u_{p-i+1}} \delta_{v_{p-k-i+1}}=8,2 \leq i \leq p-k\right\}\right|$ $=p-k-1$.

P2.6. $\left|\left\{\left(v_{i}, v_{i+k-1}\right): \delta_{v_{i}}+\delta_{v_{i+k-1}}=4, \delta_{v_{i}} \delta_{v_{i+k-1}}=4,1 \leq i \leq p-k\right\}\right|=p-k$.

P3. if $d(y, z)=p-2$ then $\left|D_{p-2}\right|=8$, we have six subsets of it:

P3.1. $\left|\left\{\left(u_{i}, u_{p+i-2}\right): \delta_{u_{i}}+\delta_{u_{p+i-2}}=6, \delta_{u_{i}} \delta_{u_{p+i-2}}=8, i=1,2\right\}\right|=2$.

P3.2. $\left|\left\{\left(u_{1}, v_{p-2}\right): \delta_{u_{1}}+\delta_{v_{p-2}}=4, \delta_{u_{1}} \delta_{v_{p-2}}=4\right\}\right|=1$.

P3.3. $\left|\left\{\left(u_{p}, v_{2}\right): \delta_{u_{p}}+\delta_{v_{2}}=4, \delta_{u_{p}} \delta_{v_{2}}=4\right\}\right|=1$.

P3.4. $\left|\left\{\left(u_{2}, v_{p-1}\right): \delta_{u_{2}}+\delta_{v_{p-1}}=6, \delta_{u_{2}} \delta_{v_{p-1}}=8\right\}\right|=1$.

P3.5. $\left|\left\{\left(u_{p-1}, v_{1}\right): \delta_{u_{p-1}}+\delta_{v_{1}}=6, \delta_{u_{p-1}} \delta_{v_{1}}=8\right\}\right|=1$.

P3.6. $\left|\left\{\left(v_{i}, v_{p+i-3}\right): \delta_{v_{i}}+\delta_{v_{p+i-3}}=4, \delta_{v_{i}} \delta_{v_{p+i-3}}=4, i=1,2\right\}\right|=2$.

P4. if $d(y, z)=p-1$ then $\left|D_{p-1}\right|=4$, we have four subsets of it:

P4.1. $\left|\left\{\left(u_{1}, u_{p}\right): \delta_{u_{1}}+\delta_{u_{p}}=4, \delta_{u_{1}} \delta_{u_{p}}=4\right\}\right|=1$.

P4.2. $\left|\left\{\left(u_{1}, v_{p-1}\right): \delta_{u_{1}}+\delta_{v_{p-1}}=4, \delta_{u_{1}} \delta_{v_{p-1}}=4\right\}\right|=1$.

P4.3. $\left|\left\{\left(u_{p}, v_{1}\right): \delta_{u_{p}}+\delta_{v_{1}}=4, \delta_{u_{p}} \delta_{v_{1}}=4\right\}\right|=1$.

P4.4. $\left|\left\{\left(v_{1}, v_{p-1}\right): \delta_{v_{1}}+\delta_{v_{p-1}}=4, \delta_{v_{1}} \delta_{v_{p-1}}=4\right\}\right|=1$.

From P1 - P4, we have:

$$
\begin{aligned}
& S c\left(P_{p}^{c} ; x\right)=4(5 p-7) x+8 \sum_{k=2}^{p-3}(3 p-3 k-1) x^{k}+40 x^{p-2}+16 x^{p-1} . \\
& \stackrel{*}{S c}\left(P_{p}^{c} ; x\right)=8(4 p-7) x+12 \sum_{k=2}^{p-3}(3 p-3 k-2) x^{k}+48 x^{p-2}+16 x^{p-1} .
\end{aligned}
$$

Corollary2.4.2: For $p \geq 5$, then:
1) $\operatorname{Sc}\left(P_{p}^{c}\right)=4(p+1)(p-1)^{2}$.
2) $S_{c}^{*}\left(P_{p}^{c}\right)=6 p(p-1)^{2}$.

Corollary2.4.3: For $p \geq 5$, then:

1) $10 \frac{2}{3} \leq \overline{S c\left(P_{p}^{c}\right)} \leq 2 p+1$.

2) $13 \frac{1}{3} \leq \overline{S^{*}\left(P_{p}^{c}\right)}<3(2 p-1) / 2$.

\section{Remark 2.4.4:}
1) $\operatorname{Sc}\left(P_{3}^{c} ; x\right)=32 x^{1}+16 x^{2}, \operatorname{Sc}\left(P_{4}^{c} ; x\right)=52 x^{1}+40 x^{2}+16 x^{3}$.
2) $S_{S c}^{*}\left(P_{3}^{c} ; x\right)=40 x^{1}+16 x^{2}, \quad S_{C}^{*}\left(P_{4}^{c} ; x\right)=72 x^{1}+48 x^{2}+16 x^{3}$. 


\subsection{Definition}

A Cog-Cycle is a graph $C_{p}^{c}, p \geq 3$ obtained from a cycle graph $C_{p}=\left\{u_{1}, u_{2}, \cdots, u_{p}, u_{1}\right\}$ with $p$ additional vertices $\left\{v_{1}, v_{2}, \cdots, v_{p}\right\}$, and edges $\left\{v_{i} u_{i}, v_{i} u_{i+1}: i=1,2, \cdots, p,\left(u_{1} \equiv u_{p+1}\right)\right\}$ as shown in Figure 5 .

It's clear that $p\left(C_{p}^{c}\right)=2 p, q\left(C_{p}^{c}\right)=3 p$, and

$$
\operatorname{diam} C_{p}^{c}=\left[\begin{array}{l}
(p / 2)+1, p \text { is even } p \geq 4 \\
(p+1) / 2, p \text { is odd } p \geq 3
\end{array}\right.
$$

Theorem 2.5.1: For $p \geq 6$, then:

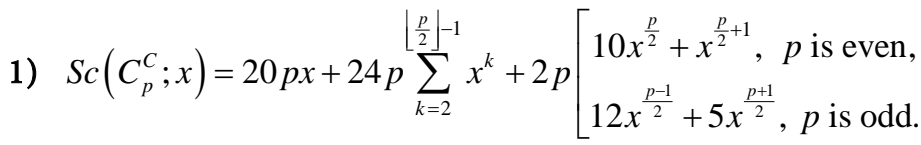
2) $S^{*} c\left(C_{p}^{c} ; x\right)=32 p x+36 p \sum_{k=2}^{\left\lfloor\frac{p}{2}\right\rfloor-1} x^{k}+2 p\left[\begin{array}{c}14 x^{\frac{p}{2}}+x^{\frac{p}{2}+1}, p \text { is even, } \\ 18 x^{\frac{p-1}{2}}+6 x^{\frac{p+1}{2}}, p \text { is odd. }\end{array}\right.$

Proof: For every vertice $y, z \in V\left(C_{p}^{c}\right)$, there is $d(y, z)=k, 1 \leq k \leq\left\lfloor\frac{p}{2}\right\rfloor+1$, and obviously $\sum_{i=1}^{\left\lfloor\frac{p}{2} \mid+1\right.}\left|D_{i}\right|=p(2 p-1)$. We will four partitions for proof:

P1. if $d(y, z)=1$, then $\left|D_{i}\right|=3 p$ and is equal to $q\left(C_{p}^{c}\right)$. We have three subsets of it:

P1.1.

$\left|\left(u_{i}, u_{i+1}\right): u_{i} u_{i+1} \in E\left(C_{p}^{c}\right), \delta_{u_{i}}+\delta_{u_{i+1}}=8, \delta_{u_{i}} \delta_{u_{i+1}}=16,1 \leq i \leq p,\left(u_{p+1} \equiv u_{1}\right)\right|=p$.

P1.2. $\left|\left\{\left(u_{i}, v_{i}\right): u_{i} v_{i} \in E\left(C_{p}^{c}\right), \delta_{u_{i}}+\delta_{v_{i}}=6, \delta_{u_{i}} \delta_{v_{i}}=8,1 \leq i \leq p\right\}\right|=p$.

P1.3.

$\left|\left\{\left(u_{i+1}, v_{i}\right): u_{i+1} v_{i} \in E\left(C_{p}^{c}\right), \delta_{u_{i+1}}+\delta_{v_{i}}=6, \delta_{u_{i+1}} \delta_{v_{i}}=8,1 \leq i \leq p,\left(u_{p+1} \equiv u_{1}\right)\right\}\right|=p$

P2. If $d(y, z)=k, 2 \leq k \leq\left\lfloor\frac{p}{2}\right\rfloor-1$, then $\sum_{k=2}^{\left\lfloor\frac{p}{2} \mid-1\right.}\left|D_{k}\right|=4 p$. We have four subsets of it:

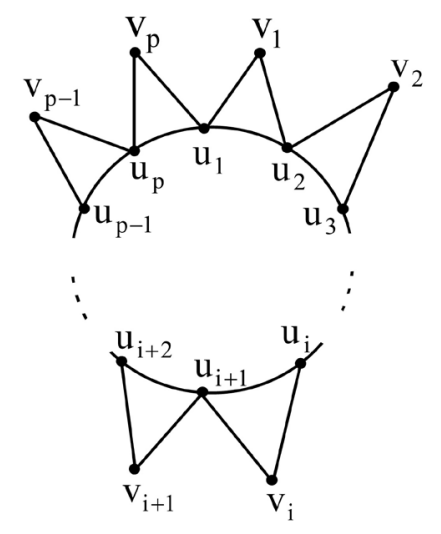

Figure 5. A Cog-Cycle Graph $C_{p}^{c}$. 
when $u_{i}$ moving to $v_{j}$ clockwise.

P2.1. $\left|\left\{\left(u_{i}, u_{j}\right): \delta_{u_{i}}+\delta_{u_{j}}=8, \delta_{u_{i}} \delta_{u_{j}}=16,1 \leq i, j \leq p,|i-j|=k, p-k\right\}\right|=p$.

P2.2. $\left|\left\{\left(u_{i}, v_{j}\right): \delta_{u_{i}}+\delta_{v_{j}}=6, \delta_{u_{i}} \delta_{v_{j}}=8,1 \leq i, j \leq p,|i-j|=k-1, p-k+1\right\}\right|=p$.

when $u_{i}$ moving to $v_{j}$ reversed clockwise.

P2.3. $\left|\left\{\left(u_{i}, v_{j}\right): \delta_{u_{i}}+\delta_{v_{j}}=6, \delta_{u_{i}} \delta_{v_{j}}=8,1 \leq i, j \leq p,|i-j|=k, p-k\right\}\right|=p$.

P2.4. $\left|\left\{\left(v_{i}, v_{j}\right): \delta_{v_{i}}+\delta_{v_{j}}=4, \delta_{v_{i}} \delta_{v_{j}}=4,1 \leq i, j \leq p,|i-j|=k-1, p-k+1\right\}\right|=p$.

P3. If $d(y, z)=\lfloor p / 2\rfloor$, when $p$ is even, then $\left|D_{p / 2}\right|=7 p / 2$, we have four subsets of it:

P3.1. $\left|\left\{\left(u_{i}, u_{i+p / 2}\right): \delta_{u_{i}}+\delta_{u_{p / 2}}=8, \delta_{u_{i}} \delta_{u_{p / 2}}=16,1 \leq i \leq p / 2\right\}\right|=p / 2$.

when $u_{i}$ moving to $v_{j}$ clockwise.

$$
\mid\left\{\left(u_{i}, v_{j}\right): \delta_{u_{i}}+\delta_{v_{j}}=6, \delta_{u_{i}} \delta_{v_{j}}=8,1 \leq i \leq 1+(p / 2)+1, j=(p / 2)+i-1\right\}
$$

P3.2. $\bigcup\left\{\left(u_{i}, v_{j}\right): \delta_{u_{i}}+\delta_{v_{j}}=6, \delta_{u_{i}} \delta_{v_{j}}=8,(p / 2)+2 \leq i \leq p, j=i-(p / 2)-1\right\} \mid$

$$
=p \text {. }
$$

when $u_{i}$ moving to $v_{j}$ reversed clockwise.

$$
\mid\left\{\left(u_{i}, v_{j}\right): \delta_{u_{i}}+\delta_{v_{j}}=6, \delta_{u_{i}} \delta_{v_{j}}=8,1 \leq i \leq p / 2, j=(p / 2)+i\right\}
$$

P3.3. $\cup\left\{\left(u_{i}, v_{j}\right): \delta_{u_{i}}+\delta_{v_{j}}=6, \delta_{u_{i}} \delta_{v_{j}}=8,1+(p / 2) \leq i \leq p, j=i-(p / 2)\right\} \mid$

$$
\begin{aligned}
& =p . \\
& \left\{\left\{\left(v_{i}, v_{j}\right): \delta_{v_{i}}+\delta_{v_{j}}=4, \delta_{v_{i}} \delta_{v_{j}}=4,1 \leq i \leq(p / 2)+1, j=i+(p / 2)-1\right\}\right.
\end{aligned}
$$

P3.4. $\cup\left\{\left(v_{i}, v_{j}\right): \delta_{v_{i}}+\delta_{v_{j}}=4, \delta_{v_{i}} \delta_{v_{j}}=4,2+(p / 2) \leq i \leq p, j=i-(p / 2)-1\right\} \mid$

$$
=p \text {. }
$$

when $p$ is odd, then $\left|D_{(p-1) / 2}\right|=4 p$, we have four subsets of it:

P'3.1. $\left.\mid\left\{\left(u_{i}, u_{j}\right): \delta_{u_{i}}+\delta_{u_{j}}=8, \delta_{u_{i}} \delta_{u_{j}}=16,1 \leq i, j \leq p,|i-j|=\mid \frac{p}{2}\right\rfloor,\left\lceil\frac{p}{2}\right\rceil\right\} \mid=p$.

when $u_{i}$ moving to $v_{j}$ clockwise.

$\left\{\left(u_{i}, v_{j}\right): \delta_{u_{i}}+\delta_{v_{j}}=6, \delta_{u_{i}} \delta_{v_{j}}=8,1 \leq i \leq(p+3) / 2, j=i+(p-3) / 2\right\}$

P'3.2. $\bigcup\left\{\left(u_{i}, v_{j}\right): \delta_{u_{i}}+\delta_{v_{j}}=6, \delta_{u_{i}} \delta_{v_{j}}=8,(p+5) / 2 \leq i \leq p, j=i-(p+3) / 2\right\} \mid$ $=p$.

when $u_{i}$ moving to $v_{j}$ reversed clockwise.

$\left\{\left\{\left(u_{i}, v_{j}\right): \delta_{u_{i}}+\delta_{v_{j}}=6, \delta_{u_{i}} \delta_{v_{j}}=8,1 \leq i \leq(p-1) / 2, j=i+(p+1) / 2\right\}\right.$

P'3.3. $\bigcup\left\{\left(u_{i}, v_{j}\right): \delta_{u_{i}}+\delta_{v_{j}}=6, \delta_{u_{i}} \delta_{v_{j}}=8,(p+1) / 2 \leq i \leq p, j=i-(p-1) / 2\right\} \mid$ $=p$.

$\left\{\left(v_{i}, v_{j}\right): \delta_{v_{i}}+\delta_{v_{j}}=4, \delta_{v_{i}} \delta_{v_{j}}=4,1 \leq i \leq(p+3) / 2, j=i+(p-3) / 2\right\}$

P'3.4. $\cup\left\{\left(v_{i}, v_{j}\right): \delta_{v_{i}}+\delta_{v_{j}}=4, \delta_{v_{i}} \delta_{v_{j}}=4,(p+5) / 2 \leq i \leq p, j=i-(p-3) / 2\right\}$

$=p$. 
P4. If $d(y, z)=\lfloor p / 2\rfloor+1$, when $p$ is even then $\left|D_{1+p / 2}\right|=p / 2$, we have:

$$
\left|\left\{\left(v_{i}, v_{i+p / 2}\right): \delta_{v_{i}}+\delta_{v_{i+p / 2}}=4, \delta_{v_{i}} \delta_{v_{i+p / 2}}=4,1 \leq i \leq p / 2\right\}\right|=p / 2 \text {. }
$$

when $p$ is odd then $\left|D_{(p+1) / 2}\right|=2 p$, we have two subsets of it:

$$
\left\{\left(u_{i}, v_{j}\right): \delta_{u_{i}}+\delta_{v_{j}}=6, \delta_{u_{i}} \delta_{v_{j}}=8,1 \leq i \leq(p+1) / 2, j=i+(p-1) / 2\right\}
$$

P4.1. $\bigcup\left\{\left(u_{i}, v_{j}\right): \delta_{u_{i}}+\delta_{v_{j}}=6, \delta_{u_{i}} \delta_{v_{j}}=8,(p+3) / 2 \leq i \leq p, j=i-(p+1) / 2\right\} \mid$

$$
\begin{aligned}
& =p . \\
& \mid\left\{\left(v_{i}, v_{j}\right): \delta_{v_{i}}+\delta_{v_{j}}=4, \delta_{v_{i}} \delta_{v_{j}}=4,1 \leq i \leq(p+1) / 2, j=i+(p-1) / 2\right\}
\end{aligned}
$$

P4.2. $\bigcup\left\{\left(v_{i}, v_{j}\right): \delta_{v_{i}}+\delta_{v_{j}}=4, \delta_{v_{i}} \delta_{v_{j}}=4,(p+3) / 2 \leq i \leq p, j=i-(p+1) / 2\right\} \mid$ $=p$.

From P1 - P4, we have:

$$
\begin{aligned}
& S c\left(C_{p}^{c} ; x\right)=20 p x+24 p \sum_{k=2}^{\lfloor p / 2\rfloor-1} x^{k}+2 p\left[\begin{array}{l}
10 x^{\frac{p}{2}}+x^{\frac{p}{2}+1} ; p \text { is even, } \\
12 x^{\frac{p-1}{2}}+5 x^{\frac{p+1}{2}} ; p \text { is odd. }
\end{array}\right. \\
& \stackrel{*}{*} c^{*}\left(C_{p}^{c} ; x\right)=32 p x+36 p \sum_{k=2}^{\lfloor p / 2\rfloor-1} x^{k}+2 p\left[\begin{array}{l}
14 x^{\frac{p}{2}}+x^{\frac{p}{2}+1} ; p \text { is even, } \\
18 x^{\frac{p-1}{2}}+6 x^{\frac{p+1}{2}} ; p \text { is odd. }
\end{array}\right.
\end{aligned}
$$

Corollary2.5.2: For $p \geq 6$, then:

1) $S c\left(C_{p}^{c}\right)=p\left(3 p^{2}+5 p-2\right)$.

2) $\stackrel{*}{S} c^{*}\left(C_{p}^{c}\right)=\frac{p}{2}\left[\begin{array}{ll}9 p^{2}+12 p-4 ; & p \text { is even, } \\ 9 p^{2}+12 p-5 ; & p \text { is odd. }\end{array}\right.$

Corollary2.5.3: For $p \geq 6$, then:

1) $12 \frac{4}{11} \leq \overline{S c}\left(C_{p}^{c}\right)<(3 p+7) / 2$.

2) $17.7<\stackrel{\bar{*}}{S}\left(C_{p}^{c}\right)<(18 p+53) / 8$.

Remark 2.5.4:

1) $\operatorname{Sc}\left(C_{4}^{c} ; x\right)=80 x^{1}+80 x^{2}+8 x^{3}, \operatorname{Sc}\left(C_{5}^{c} ; x\right)=100 x^{1}+120 x^{2}+50 x^{3}$.

2) $\stackrel{*}{S} c\left(C_{4}^{c} ; x\right)=128 x^{1}+112 x^{2}+8 x^{3}, \stackrel{*}{S} c\left(C_{5}^{c} ; x\right)=160 x^{1}+180 x^{2}+60 x^{3}$.

\section{Conflicts of Interest}

The authors declare no conflicts of interest regarding the publication of this paper.

\section{References}

[1] Buckley, F. and Harary, F. (1990) Distance in Graphs. Addison-Wesley, New York.

[2] Chartrand, G. and Lesniak, L. (1986) Graphs and Digraphs. 2nd Edition, Wadsworth and Brooks/Cole, Pacific Grove.

[3] Devillers, J. and Balaban, A.T. (1999) Topological Indices and Related Descriptors in QSAR and QSPR. Gordon \& Breach, Amsterdam. 
[4] Diestel, R. (2000) Graph Theory, Electronic. Springer-Verlag, New York.

[5] Diudea, M.V. (2001) QSPR/QSAR Studies by Molecular Descriptors. Nova, Huntington, New York.

[6] Diudea, M.V., Gutman, I. and Jantschi, L. (2001) Molecular Topology. Nova, Huntington, New York.

[7] Schultz, H.P. (1989) Topological Organic Chemistry 1. Graph Theory and Topological Indices of Alkanes. The Journal for Chemical Information and Computer scientists, 29, 227-228. https://doi.org/10.1021/ci00063a012

[8] Klavžar, S. and Gutman, I. (1997) Wiener Number of Vertex-Weighted Graphs and a Chemical Application. Discrete Applied Mathematics, 80, 73-81. https://doi.org/10.1016/S0166-218X(97)00070-X

[9] Ahmed, M. and Haitham, N. (2017) Schultz and Modified Schultz Polynomials of Two Operations Gutman's. International Journal of Enhanced Research in Science, Technology \& Engineering, 6, 68-74. https://doi.org/10.11648/j.acm.20170606.14

[10] Ahmed, M. and Haitham, N. (2017) Schultz and Modified Schultz Polynomials of Cog-Complete Bipartite Graphs. Applied and Computational Mathematics, 6, 259-264. https://doi.org/10.11648/j.acm.20170606.14

[11] Behmaram, A., Yousefi-Azari, H. and Ashrafi, A.R. (2011) Some New Results on Distance-Based Polynomials. MATCH Communications in Mathematical and in Computer Chemistry, 65, 39-50. 